\title{
Does Body Surface Potential Mapping (BSPM) Predict Functional Recovery in Chronic Ischemic Myocardium after Revascularization?
}

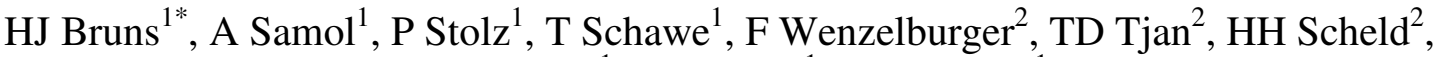 \\ G Breithardt ${ }^{1}$, T Wichter ${ }^{1}$, C Vahlhaus $^{1}$ \\ ${ }^{1}$ Department of Cardiology and Angiology, Hospital of the University of Münster, Münster, Germany \\ * HJ B. currently at CRM Research, Medtronic Bakken Research Center, Maastricht, Netherlands \\ ${ }^{2}$ Department of Thoracic and Cardiovascular Surgery, Hospital of the University of Münster, \\ Münster, Germany
}

\begin{abstract}
Background: Irreversibly dysfunctional myocardium is electrically characterized by decreased amplitudes and prolonged duration of local electrograms. We investigated whether ECGs from body surface correlate with epicardial electrograms. Methods: A 120-channel body surface potential mapping (BSPM) was performed in 24 patients before CABG. During CABG epicardial mapping was performed. For all BSPM leads the $Q$ amplitude and QR-duration were measured and correlated to epicardial electrogram characteristics. Results: $Q$-wave-amplitude neighboring the area over the left scapula correlated well with epicardial amplitudes in scarred LAD. QR-duration caudal to V5,V6 correlated well with epicardial signal duration in scarred $C x A, Q$ amplitude in paramedian epigastric region correlated with epicardial amplitudes in scarred RCA. Conclusion: Epicardial electrogram characteristics can be estimated from body surface in patients with chronic ischemia.
\end{abstract}

\section{Introduction}

The discovery of myocardial hibernation, identified in patients with coronary artery disease undergoing coronary artery bypass surgery $(1 ; 2)$, has introduced fundamental changes to the treatment of patients with left ventricular dysfunction due to coronary artery disease $(3 ; 4)$. If these patients demonstrated myocardial viability during lowdose dobutamine echocardiography, revascularization not only improved myocardial function but also survival (5). Therefore, it is of utmost relevance to detect viable regions with the potential to recover before planning the optimal treatment strategy in patients with LV dysfunction due to ischemic cardiomyopathy. Like electromechanical NOGA-mapping (6), direct epicardial mapping (DEM) has been shown to differentiate viable myocardium from myocardial infarction (7). Simpler methods are required to save time and money, to increase patients' acceptance, and to reduce potential risks of invasive procedures, such as DEM during $\mathrm{CABG}$ or NOGA-mapping during coronary angiography. Therefore, we investigated whether or not electrocardiograms from body surface (BSPM) correlate with electrograms mapped directly on the epicardial surface (DEM).

\section{Methods}

\section{Patients}

24 patients (22 male, 2 female, age: $63 \pm 8$ years, EF: $45 \pm 12 \%$ ) with coronary artery disease and elective indication for coronary artery bypass graft surgery (CABG) were prospectively enrolled in this study. The study protocol had been approved by the local Ethical Review Board of the University of Münster, Germany. Patients gave written informed consent before entering the study.

\section{Body Surface Potential Mapping}

Electrodes were applied to the chest in vertical strips (Fig.1); each electrode (Foxmed $\mathrm{GmbH}$, Idstein, Germany) had a $10 \mathrm{~mm}$ diameter $\mathrm{Ag} / \mathrm{AgCl}$ sensor embedded in an epoxy housing with a $2 \mathrm{~mm}$ gel cavity. In vertical direction, the inter-electrode distance on the strips were $50 \mathrm{~mm}$. The 120 unipolar ECG leads referred to Wilson central terminal and were recorded simultaneously. The ECG signals were amplified, band pass-filtered $(0.16-400 \mathrm{~Hz})$ and $\mathrm{A} / \mathrm{D}$ converted to 16 bit samples $(0.5 \mu \mathrm{V}$ least significant bit) using a sampling rate of $1 \mathrm{kHz}$ (Mark-6, Biosemi, Amsterdam, Netherlands). Leads, which were excessively noisy or which contained artifacts, were excluded from the data set for further off-line analysis. Measurements were performed at resting conditions for 5 minutes. 


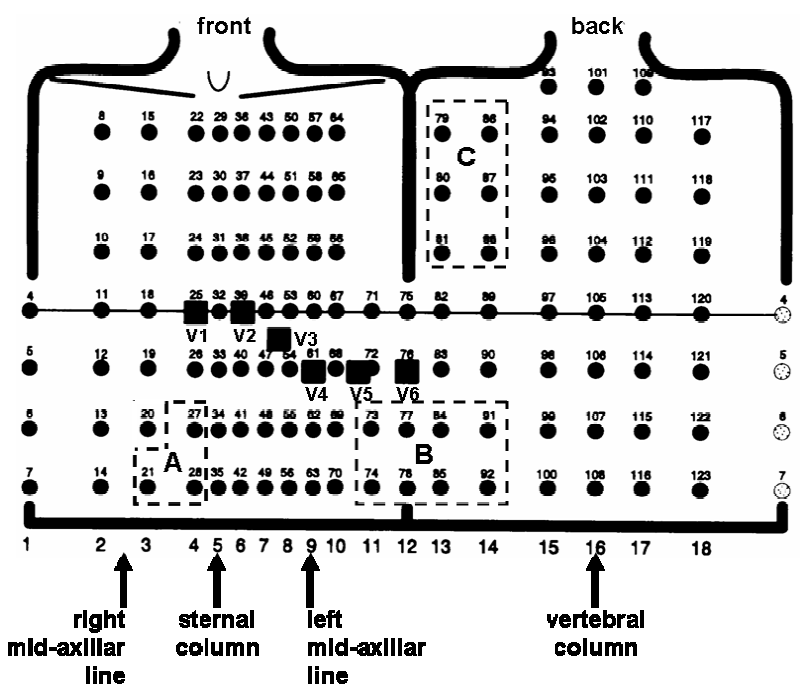

Figure 1: Scheme of Body Surface Potential Mapping (BSPM), a ECG mapping procedure covering the entire body surface and oriented on anatomical landmarks. Filled rectangles indicate position of standard pre-cordial leads V1-V6. Alternative areas of body surface A,B and C showed ECG characteristics that were linked to epicardial electrograms obtained from ischemic zones for different areas defined by coronary artery supply (LAD, CxA, RCA).

\section{Echocardiography (Echo)}

Segmental assessment of myocardial function and viability was performed using transthoracic echocardiography preoperatively and $7 \pm 2$ months (range 3-10 months) after CABG. For acquisition and digital storage of parasternal long- and short-axis and apical four-, three-, and two-chamber echocardiograms, commercially available equipment was used (3.5-MHz transducer; SONOS 5500, Agilent Technologies). Two experienced readers, blinded to the clinical results, analyzed segmental myocardial function on quad-screen views of the digitally stored echocardiograms. Based on the handmade maps made during direct epicardial mapping the pre- and post-operatively acquired echocardiography data was precisely assigned to the sight of every electrode pair in each patient. In accordance to the number of electrode pairs and strips from atrioventricular groove to apex and from anterior to inferior border of the left ventricular free wall segmental echocardiograms were evaluated. Segments were graded semiquantitatively on a 5-point scoring system (1: normal, 2: mild hypokinesis, 3: severe hypokinesis, 4: akinesis, and 5: dyskinesis). Segmental improvement was defined as a decrease in wall motion score of at least $20 \%$ (8). Improved segments of a region supplied with a bypass graft were classified as viable, preoperatively dysfunctional, revascularized segments, that did not improve were classified as non-viable or scarred.

\section{Intraoperative direct epicardial mapping (DEM)}

After opening the chest and the pericardium, the right atrium and the aorta were connected to the cardiopulmonary bypass. Epicardial mapping was performed using a ventricular jacket array which was carefully fixed around both ventricles without ignoring any part of the left ventricular free wall, especially the true posterior wall. Data were recorded with a commercial system (CardioMapp®, Prucka Engineering Inc., Houston, Texas, USA) as previously reported $(9 ; 10)$. In brief, the ventricular jacket array consisted of 102 gold-plated bipolar electrode pairs, arranged in 12 strips of printed copper layers on flexible plastic material: 6 strips with 10 pairs of electrodes and 6 strips with 7 pairs of electrodes (Figure 1). Each electrode measured $1.5 \mathrm{~mm}$ in diameter. In vertical direction, the distance between electrode pairs was $13 \mathrm{~mm}$ with an inter-pole distance of $4 \mathrm{~mm}$. In horizontal direction, the 12 electrode strips were connected with elastic fabric to fit the ventricular jacket array to different sizes of the heart and to optimize LV contact.

In each patient, a map with the exact position of the 102 electrode pairs was drawn. Atrioventricular groove and left anterior and posterior interventricular arteries served as landmarks. Those electrode pairs overhanging at apical or atrial border or positioned on fatty epicardium were marked on the map to identify all segments without LV contact. Multiple registrations of $8.9 \mathrm{~s}$ duration were performed while the patients were in stable sinus rhythm during partial normothermic cardiopulmonary bypass and before induction of ventricular fibrillation. Mechanical pulmonary ventilation was interrupted for the time of the recordings and the systolic blood pressure was set to about $100 \mathrm{mmHg}$. Signals separately band-pass filtered $(0.2-300 \mathrm{~Hz}$ ), amplified (dynamic input range $\pm 16 \mathrm{mV}$, gain 63), analogue-to-digital converted (1 kHz sampling rate, 12-bit digital resolution, $7.8 \mu \mathrm{V}$ least significant bit) and stored on a hard disk.

\section{Signal analysis (DEM)}

Signal analysis was performed offline. The recording with the highest signal-to-noise ratio without any offset variation due to respiratory motion was selected for single-beat analysis as reported before(11). Two characteristics of the local electrograms were calculated: the bipolar amplitude by measurement of peak-to-peak amplitude and the electrogram duration by a modified "Simpson" algorithm. 


\section{Signal analysis(BSPM)}

The BSPM signal analysis was also performed offline. For each BSPM lead the amplitude offset was corrected by the mean amplitude level of a $20 \mathrm{~ms}$ reference window located in the PQ interval of the ECG. For each surface lead, the minimum/maximum QRS amplitudes, the QR duration (earliest limb lead Q-wave to maximum R-wave) and the QRS integral were calculated.

\section{Statistics}

Statistical analysis was performed using SPSS software (Version 12, SPSS Inc., Chicago, Illinois, USA). Data were subjected to a one-way analysis of variance (ANOVA). Linear regression analysis between BSPM and DEM parameters was performed. All data are presented as mean $\pm \mathrm{SD}$.

\section{Results}

Q-wave-amplitude neighboring the area over the left scapula (BSPM-channels 79, 80,81,86, 87, and 88) correlated well with the local duration of electrograms recorded in scarred LAD-regions $\left(\mathrm{r}^{2}=0.6 \pm 0.08\right.$, mean $\pm S D, p<0.001$ ), Figure 2 .

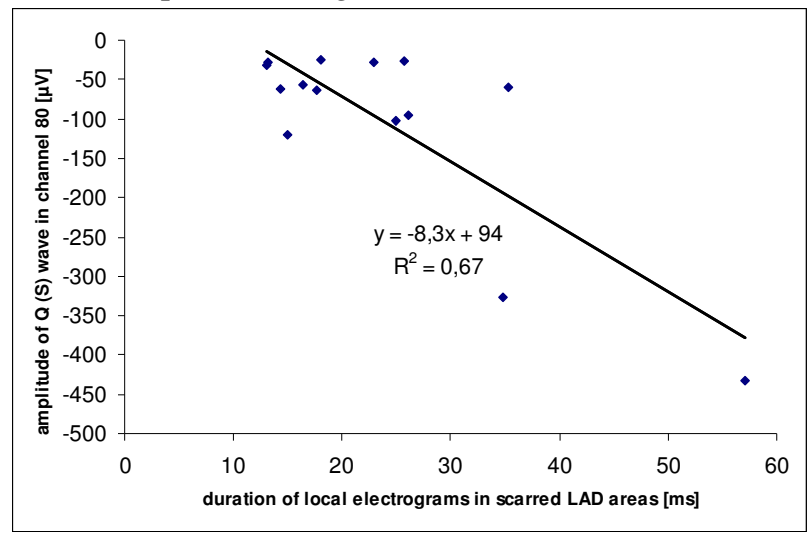

Figure 2: Comparison of BSPM amplitudes and epicardial electrogram durations in scarred LAD areas.

QR-duration in the area neighboring on the left thorax (BSPM channels 73, 74, 77, 78, 84, 85, 91, 92) correlated excellent with mean signal duration in scarred CxAregion $\left(r^{2}=0.8 \pm 0.03\right.$, mean $\left.\pm S D, p<0.001\right)$.

Q-wave-amplitude in the zone neighboring in the paramedian epigastric region (BSPM-channels 21, 27, and 28) correlated with the amplitude of electrograms in scarred RCA-region $\left(21: \mathrm{r}^{2}=0.48, \mathrm{p}<0.001 ; 27: \mathrm{r}^{2}=0.39\right.$, $\left.\mathrm{p}<0.001 ; 28: \mathrm{r}^{2}=0.44, \mathrm{p}<0.001\right)$, Figure 3 .

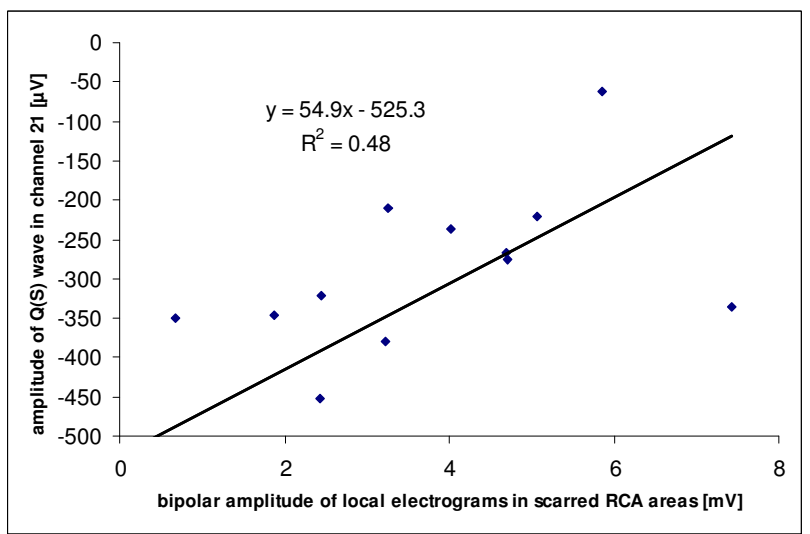

Figure 3: Comparison of BSPM amplitudes and epicardial electrogram amplitudes in scarred RCA areas.

\section{Discussion and conclusions}

The concept of "electromechanical mismatch" in chronic ischemic myocardium characterizes viable myocardium by maintained electrical properties of the myocardium but impaired function (10). Vice versa impaired electrical properties, therefore, indicate necrotic non-viable tissue segments that will not show improved function after revascularization procedures. Even without using techniques of "inverse solutions", for regions showing non-viable myocardium a link between direct epicardial electrograms and body surface mapping potentials was established. Furthermore, body surface areas that are not in the scope of standard pre-cordial lead positions V1 to V6 were identified that represent changes in ECG characteristics based on local remodelling in different left ventricular coronary artery supply areas.

In future, BSPM might be used as a non-invasive tool to predict the functional recovery of myocardium after coronary revascularization. Further studies need to be performed to confirm this and to overcome technical and procedure-related limitations of this method.

\section{Acknowledgements}

This work was supported in part by grants from the 1) Interdisciplinary Centre for Clinical Research (IZKF; B1, BMBF-01KS 9604), Münster, Germany; 2) Münster Heart Center; and 3) the German Academic Exchange Program (DAAD; 313/sf-ppp-kr) 


\section{References}

(1) Rahimtoola SH. Coronary bypass surgery for chronic angina-1981. A perspective. Circulation $1982 ; 65: 225-241$.

(2) Rahimtoola $\mathrm{SH}$. A perspective on the three large multicenter randomized clinical trials of coronary bypass surgery for chronic stable angina. Circulation 1985; 72:V123-V135.

(3) Elsasser A, Schlepper M, Klovekorn WP, Cai Wj, Zimmermann R, Muller KD et al. Hibernating myocardium: an incomplete adaptation to ischemia. Circulation 1997; 96:2920-2931.

(4) Gunning MG, Chua TP, Harrington D, Knight CJ, Burman E, Pennell DJ et al. Hibernating myocardium: clinical and functional response to revascularisation. Eur J Cardiothorac Surg 1997; 11:1105-1112.

(5) Afridi I, Grayburn PA, Panza JA, Oh JK, Zoghbi WA, Marwick TH. Myocardial viability during dobutamine echocardiography predicts survival in patients with coronary artery disease and severe left ventricular systolic dysfunction. J Am Coll Cardiol 1998; 32:921-926.

(6) Perin EC, Silva GV, Sarmento-Leite R, Sousa ALS, Howell M, Muthupillai R et al. Assessing Myocardial Viability and Infarct Transmurality With Left Ventricular Electromechanical Mapping in Patients With Stable Coronary Artery Disease: Validation by Delayed-Enhancement Magnetic Resonance Imaging. Circulation 2002; 106:957961.

(7) Vahlhaus C, Bruns HJ, Stypmann J, Tjan DT, Janssen F, Schäfers M et al. Direct epicardial mapping predicts the recovery of left ventricular dysfunction in chronic ischaemic myocardium. European Heart Journal 2004; 25:151-157.

(8) Dhar SC, Birnbaum Y, Hayes S, Naqvi T, Cercek $\mathrm{B}$, Blanche $\mathrm{C}$ et al. Milrinone echocardiographic viability test: a pilot study. J Am Soc Echocardiogr 2001; 14:668-675.

(9) Vahlhaus C, Schäfers M, Bruns HJ, Janssen F, Stypmann J, Hammel D et al. Direct epicardial mapping can differentiate hibernating from scarred myocardium: a validation study with $18 \mathrm{~F}-\mathrm{FDG}-$ PET. Ann Noninvasive Electrocardiol 2002; 7:349-356.

(10) Bruns HJ, Janssen F, Schäfers M, Hammel D, Fetsch T, Arslan O et al. Signal characteristics of multichannel epicardial electrograms in chronic ischaemic and scarred myocardium: electromechanical mismatch indicates viability in regions of myocardial dysfunction. Basic Res Cardiol 2001; 96:98-105.
(11) Bruns HJ, Eckardt L, Vahlhaus C, Schulze-Bahr E, Haverkamp W, Borggrefe M et al. Body surface potential mapping in patients with Brugada syndrome: right precordial ST segment variations and reverse changes in left precordial leads. Cardiovasc Res 2002; 54:58-66.

\author{
Address for correspondence: \\ Hans-Jürgen Bruns, PhD \\ CRM Research \\ Medtronic Bakken Research Center \\ Endepolsdomein 5 \\ 6229 GW Maastricht \\ The Netherlands \\ Phone: +31 433566861 \\ e-mail: hans-juergen.bruns@medtronic.com
}

